International Journal of Bifurcation and Chaos, Vol. 21, No. 5 (2011) 1457-1467

(C) World Scientific Publishing Company

DOI: $10.1142 / \mathrm{S} 0218127411029148$

\title{
THE SPECTRUM OF CHAOTIC TIME SERIES (II): WAVELET ANALYSIS
}

\author{
GOONG CHEN* \\ Department of Mathematics, Texas A\&M University, \\ College Station, TX 77843-3368, USA \\ and \\ Science Program, Texas A\&M University at Qatar \\ Doha, Qatar \\ gchen@math.tamu.edu \\ SZE-BI HSU \\ Department of Mathematics, National Tsing Hua University, \\ No. 101, Sec. 2, Guangfu Rd., Hsinchu, Taiwan 300, R.O.C. \\ sbhsu@math.nthu.edu.tw \\ YU HUANG* \\ Department of Mathematics, Sun Yat-sen (Zhongshan) University, \\ No. 135 Xingang Road West, Guangzhou 510275, P. R. China \\ stshyu@mail.sysu.edu.cn \\ MARCO A. ROQUE-SOL \\ Department of Mathematics, Texas A\&M University, \\ College Station, TX 77843-3368, USA \\ roquesol@math.tamu.edu
}

Received April 6, 2010; Revised October 13, 2010

\begin{abstract}
This paper is a continuation of Part I where the authors treated the Fourier analysis of chaotic time series generated by a chaotic interval map. Here, we perform multiresolution analysis by using wavelet coefficients and characterize some necessary and sufficient conditions for the occurrence of chaos by the exponential growth with respect to the number of iterations $n$ of certain sums of the wavelet coefficients.
\end{abstract}

Keywords: Topological entropy; total variation; chaos; wavelet; multiresolution analysis.

\section{Introduction}

This paper is a continuation of Part I [Chen et al., 2011]. Here, again we analyze the spectrum of a time series comprised of the iterates of a deterministic chaotic map, but by using wavelets.

Wavelet transforms use a waveform ("small wave", the mother wavelet function $\psi(t)$ ) which is scaled and translated to process, reconstruct and match the input signal ([Dai, 2006; Daubechies, 1992; Meyer \& Salinger, 1995]). Such transforms can be classified into three types: the continuous wavelet transform (CWT), the discretized wavelet transform (DWT) and multiresolution-based wavelet transform (MRA, for multiresolution analysis). In comparison with the Fourier transform, in which signals are represented as an integral or sum of real or complex sinusoidals and the transform is only localized in frequency, wavelets are localized in both time and frequency. In a sense, CWT may be likened to the continuous Fourier transform, and

\footnotetext{
*Work was completed while visiting Center for Theoretical Sciences, National Tsing Hua University, Hsinchu, Taiwan.
} 
DWT may be to the Fourier series. But MRA adds an extra auxiliary function, the father wavelet function $\phi(t)$, constituting the basis for the algorithm of the fast wavelet transform. Computationally, for a data set of size $N$, the complexity of the discrete wavelet transform takes $O(N)$ time as compared to $O(N \log N)$ for the fast Fourier transform. In practical applications, for the sake of efficiency, one often prefers continuously differentiable functions with compact support as (prototype) mother wavelet functions. During the past two decades, we have seen a vast number of applications of wavelets to signal and image processing, that often supercedes the conventional Fourier transform. Thus, it is not surprising to see earlier work such as [Azad \& Sett, 2003; Perman \& Hamilton, 1992] in an attempt to apply wavelets to the analysis of chaos. Nevertheless, rigorous mathematical results are totally lacking, to the best of our knowledge. This motivates our work in this paper.

In this paper, in Sec. 2 we first review some basic properties of wavelet transforms and notation as prerequisites for the subsequent development. In Sec. 3, we link the wavelet transform with the total variation of a map via the Fourier transform and the Parseval identity (Theorem 1). We then further derive necessary and sufficient conditions for chaos in terms of MRA coefficients in Corollary 3.2. Such conditions, determined by whether certain sums of the wavelet coefficients grow exponentially with respect to $n$ (i.e. the number of iterations), have a good deal of similarities with the main results we obtained earlier in Part I [Chen et al., 2011] based on the Fourier analysis. In Sec. 4, using Haar wavelets we have demonstrated that the theorems we have obtained in Sec. 3 are quite tight.

\section{Prerequisite: Notations and Properties of Wavelets}

We inherit the theoretical background from Part I [Chen et al., 2011]. Now, for $f \in L^{1}(\mathbb{R})$, define its Fourier transform by

$$
\hat{f}(\omega)=\int_{\mathbb{R}} e^{-i 2 \pi \omega t} f(t) d t .
$$

Then by the denseness of $L^{1}(\mathbb{R}) \cap L^{2}(\mathbb{R})$ in $L^{2}(\mathbb{R})$, we can define $\hat{f}$ for any $f \in L^{2}(\mathbb{R})$ by the usual continuity argument. From this, we have the Parseval identity

$$
\langle f, g\rangle=\langle\hat{f}, \hat{g}\rangle, \quad f, g \in L^{2}(\mathbb{R})
$$

and the Plancherel formula

$$
\|f\|=\|\hat{f}\|, \quad f \in L^{2}(\mathbb{R}),
$$

where $\langle$,$\rangle and \|\cdot\|$ in (2) and (3) denote, respectively, the $L^{2}$-inner product and the $L^{2}$-norm. A function $\psi$ in $L^{2}(\mathbb{R})$ is called a (mother) wavelet if $\psi$ satisfies the admissible condition:

$$
C_{\psi}=\int_{\mathbb{R}} \frac{|\hat{\psi}(\omega)|^{2}}{|\omega|} d \omega<+\infty
$$

If, in addition, $\psi \in L^{1}(\mathbb{R})$ then $\hat{\psi}$ is continuous and thus (4) implies

$$
\int_{-1}^{1} \frac{|\hat{\psi}(\omega)|^{2}}{|\omega|} d \omega<+\infty
$$

further implying $\hat{\psi}(0)=0$. That is,

$$
\int_{\mathbb{R}} \psi(t) d t=0 .
$$

For a given mother wavelet $\psi$, we can generate a doubly-indexed family of wavelets by dilation and translation:

$$
\psi_{s, b}(t)=\frac{1}{\sqrt{|s|}} \psi\left(\frac{t-b}{s}\right) .
$$

Definition 2.1. Let $\psi$ be a mother wavelet and $f \in L^{2}(\mathbb{R})$. The continuous wavelet transform of $f$ with respect to $\psi$ is given by

$$
\begin{aligned}
(W f)(s, b) & =\left\langle f, \psi_{s, b}\right\rangle \\
& =\int_{\mathbb{R}} f \frac{1}{\sqrt{|s|}} \overline{\left(\frac{t-b}{s}\right)} d t .
\end{aligned}
$$

A function $\psi \in L^{2}(\mathbb{R})$ is said to be a dyadic wavelet if the following stability condition holds: there exist two positive constants $A, B$ such that

$$
A \leq \sum_{j}\left|\hat{\psi}\left(2^{-j} \omega\right)\right|^{2} \leq B, \quad \text { a.e. } \omega \text {. }
$$

It is easy to see that the stability condition (7) implies the admissible condition (4). More precisely, we have the following.

Lemma 1. Let $\psi$ be a dyadic wavelet, i.e. condition (7) holds. Then

$$
A \ln 2 \leq \int_{0}^{\infty} \frac{|\hat{\psi}(\omega)|^{2}}{|\omega|} d \omega, \quad \int_{-\infty}^{0} \frac{|\hat{\psi}(\omega)|^{2}}{|\omega|} d \omega \leq B \ln 2 .
$$


Moreover, if $A=B$ in (7), then

$$
C_{\psi}=\int_{\mathbb{R}} \frac{|\hat{\psi}(\omega)|^{2}}{|\omega|} d \omega=2 A \ln 2 .
$$

Definition 2.2. Let $\psi$ be a dyadic wavelet and $f \in L^{2}(\mathbb{R})$. The dyadic wavelet transform of $f$ with respect to $\psi$ is defined as

$$
\begin{aligned}
\left(W_{j} f\right)(b) & =\left\langle f, 2^{j / 2} \psi\left(2^{j}(\cdot-b)\right)\right\rangle \\
& =2^{j / 2} \int_{\mathbb{R}} f(t) \overline{\psi\left(2^{j}(t-b)\right)} d t .
\end{aligned}
$$

Computationally, it is impossible to analyze a signal using all coefficients from the continuous wavelet transform (6). Thus, a discretized wavelet transform is in order. It is of great advantage to have a mother wavelet $\psi$ such that

$$
\left\{\psi_{j, n}(t)=2^{j / 2} \psi\left(2^{j} t-n\right)\right\}_{(j, n) \in Z^{2}}
$$

forms an orthonormal basis in $L^{2}(\mathbb{R})$. Multiresolution analysis (MRA) is an effective approach for constructing such a basis. MRA consists of a sequence of closed subspaces $V_{j}$ of $L^{2}(\mathbb{R})$ with the following conditions

(1) $\cdots \subset V_{-2} \subset V_{-1} \subset V_{0} \subset V_{1} \subset V_{2} \subset \cdots$;

(2) $\overline{\bigcup_{j} V_{j}}=L^{2}(\mathbb{R}), \bigcap_{j} V_{j}=\{0\}$;

(3) $f \in V_{j} \Leftrightarrow f(2 \cdot) \in V_{j+1}$;

(4) $f \in V_{0} \Leftrightarrow f(\cdot-k) \in V_{0}, \forall k \in Z$;

(5) There exists a $\varphi \in V_{0}$ such that $\{\varphi(\cdot-k)\}_{k}$ is an orthonormal basis for $V_{0}$.

Such function $\varphi$ is said to be a scaling function or a father wavelet.

For a given MRA, a standard way to construct an orthonormal basis in $L^{2}(\mathbb{R})$ is given in [Daubechies, 1992, Theorem 5.1.1].

Lemma 2. If a ladder of closed subspaces $\left\{V_{j}\right\}_{j \in Z}$ in $L^{2}(\mathbb{R})$ satisfies conditions (1)-(5) above, then there exists an associated orthonormal wavelet basis $\left\{\psi_{j, k} \mid j, k \in Z\right\}$ for $L^{2}(\mathbb{R})$ such that

$$
P_{j+1}=P_{j}+\sum_{k}\left\langle\cdot, \psi_{j, k}\right\rangle \psi_{j, k},
$$

where $P_{j}$ is the orthogonal projection onto $V_{j}$. A feasible way to construct the wavelet $\psi$ is by its Fourier transform

$$
\hat{\psi}(\omega)=e^{-i \pi \omega} \overline{m_{0}(\pi(\omega+1))} \hat{\varphi}\left(\frac{\omega}{2}\right),
$$

where $m_{0}$ is a periodic function (with period $2 \pi$ ) called the faltering function, given by

$$
m_{0}(\omega)=\frac{1}{2} \sum_{k} h_{k} e^{-i k \omega},
$$

where $\left\{h_{k}\right\}$ is determined uniquely by the representation of $\varphi$ :

$$
\varphi(t)=\sum_{k} h_{k} \varphi(2 t-k) .
$$

(This decomposition is unique since $\{\varphi(2 \cdot-k)\}_{k}$ is an orthonormal basis for $V_{1}$ by the assumptions.)

Similarly, the function $\psi$ given by (10) has the decomposition

$$
\psi(t)=\sum_{k} g_{k} \varphi(2 t-k),
$$

where

$$
g_{k}=(-1)^{k-1} \bar{h}_{1-k} .
$$

It follows from Lemma 2 that each $f \in L^{2}(\mathbb{R})$ has two representations: the first is

$$
f(t)=\sum_{j, k \in Z} d_{j, k} \psi_{j, k}(t),
$$

and the second is, for any $j_{0} \in Z$,

$$
f(t)=\sum_{k \in Z} c_{j_{0}, k} \varphi_{j_{0}, k}(t)+\sum_{j \geq j_{0}} \sum_{k \in Z} d_{j, k} \psi_{j, k}(t),
$$

where

$$
c_{j, k}=\left\langle f, \varphi_{j, k}\right\rangle, \quad d_{j, k}=\left\langle f, \psi_{j, k}\right\rangle .
$$

Also an MRA leads naturally to a fast scheme for the computation of the wavelet coefficients $c_{j, k}$ and $d_{j, k}$ important in applications. From (11), we have

$$
\begin{aligned}
c_{j, k} & =\int f(t) 2^{\frac{j}{2}} \overline{\varphi\left(2^{j} t-k\right)} d t \\
& =\sum_{l} \bar{h}_{l} \int f(t) 2^{\frac{j}{2}} \overline{\varphi\left(2^{j+1} t-2 k-l\right)} d t \\
& =\frac{1}{\sqrt{2}} \sum_{l} \bar{h}_{l} \int f(t) \overline{\varphi_{j+1,2 k+l}(t)} d t .
\end{aligned}
$$

So we have a fast scheme for the decomposition computation:

$$
c_{j, k}=\frac{1}{\sqrt{2}} \sum_{l} \bar{h}_{l-2 k} c_{j+1, l} .
$$

Similarly, from (12), we have

$$
d_{j, k}=\frac{1}{\sqrt{2}} \sum_{l} \bar{g}_{l-2 k} c_{j+1, l} .
$$


On the other hand, let $W_{j}$ be the orthogonal complement of $V_{j}$ in $V_{j+1}$. That is, $V_{j+1}=V_{j} \oplus W_{j}$. Since $\left\{\varphi_{j, l}\right\}_{l \in Z}$ and $\left\{\psi_{j, l}\right\}_{l \in Z}$ are orthonormal bases in $V_{j}$ and $W_{j}$, respectively, $\varphi_{j+1, k} \in V_{j+1}$ can be decomposed uniquely as

$$
\varphi_{j+1, k}(t)=\sum_{l}\left[a_{k, l} \varphi_{j, l}(t)+b_{k, l} \psi_{j, l}(t)\right],
$$

where, by orthonormality,

$$
\begin{aligned}
a_{k, l}= & \int \overline{\varphi_{j, l}(t)} \varphi_{j+1, k}(t) d t \\
= & 2^{j+1 / 2} \int \overline{\varphi\left(2^{j} t-l\right)} \varphi\left(2^{j+1} t-k\right) d t \\
= & 2^{j+1 / 2} \sum_{m} \bar{h}_{m} \int \overline{\varphi\left(2^{j+1} t-2 l-m\right)} \\
& \times \varphi\left(2^{j+1} t-k\right) d t \\
= & \frac{1}{\sqrt{2}} \sum_{m} \bar{h}_{m} \int \overline{\varphi(t-2 l-m)} \varphi(t-k) d t \\
= & \frac{1}{\sqrt{2}} \sum_{m} \bar{h}_{m} \delta_{2 l+m, k}=\frac{1}{\sqrt{2}} \bar{h}_{k-2 l} .
\end{aligned}
$$

Similarly,

$$
b_{k, l}=\frac{1}{\sqrt{2}} \bar{g}_{k-2 l} .
$$

Substituting the above two equations into (17) and then taking the inner product with $f$, we have

$$
c_{j+1, k}=\frac{1}{\sqrt{2}} \sum_{l}\left(h_{k-2 l} c_{j . l}+g_{k-2 l} d_{j, l}\right) .
$$

Formula (18) is called the fast scheme for reconstruction computation.

\section{Detecting Chaos with Wavelets}

Having furnished the wavelet prerequisite, we now enter the main section for the study of chaotic time series by wavelets. In what follows, we always assume that $f: I=[0,1] \rightarrow I$ is continuous and piecewise monotone with finitely many extremal points. We take $f$ as a function on the whole line $\mathbb{R}$ by extending $f(t)=0$ for all $t \notin I$. Thus $f \in L^{2}(\mathbb{R}) \cap L^{1}(\mathbb{R})$. Its Fourier transform is

$$
\hat{f}(w)=\int_{\mathbb{R}} f(t) e^{-i 2 \pi w t} d t=\int_{0}^{1} f(t) e^{-i 2 \pi w t} d t .
$$

As in Part I [Chen et al., 2011], the total variation $V_{I}(f)$ of $f$ on $I$ is defined as before to be the supremum of all sums

$$
\sum_{k=1}^{m}\left|f\left(t_{k}\right)-f\left(t_{k-1}\right)\right|
$$

with respect to all partitions $\left\{t_{k}\right\}_{k=0}^{m}$ on $[0,1]$ such that $0=t_{0}<t_{1}<\cdots<t_{m}=1$. In brevity, we write $V_{I}(f)$ as $V(f)$. The following lemma is a version of [Edwards, 1979, Theorem 2.3.6].

Lemma 3. Let $f: I \rightarrow I$ be continuous with bounded total variation. Then

$$
2 \pi|\omega||\hat{f}(\omega)| \leq 2+V(f), \quad \forall \omega \in \mathbb{R} .
$$

Proof. If $\omega=0,(20)$ holds obviously. Assume $w \neq 0$, then one may write (19) as

$$
\hat{f}(\omega)=\int_{0}^{1} f(t) d\left[\frac{e^{-i 2 \pi \omega t}}{-i 2 \pi \omega}\right] .
$$

Set

$$
g(t)=\frac{e^{-i 2 \pi \omega t}}{-i 2 \pi \omega}
$$

From the definition of an integral, for any given $\varepsilon>0$, there exists a sufficiently fine partition $0=t_{0}<t_{1}<\cdots<t_{m}=1$ of the interval $[0,1]$, such that

$$
\left|\hat{f}(\omega)-\sum_{k=1}^{m} f\left(t_{k}\right)\left[g\left(t_{k}\right)-g\left(t_{k-1}\right)\right]\right|<\varepsilon .
$$

Denoting by $\sum$ the sum appearing within the absolute value signs above, and applying partial summation, we obtain

$$
\begin{aligned}
\sum= & {\left[f(1) g(1)-f\left(t_{1}\right) g(0)\right] } \\
& -\sum_{k=1}^{m-1}\left[f\left(t_{k+1}\right)-f\left(t_{k}\right)\right] g\left(t_{k}\right) .
\end{aligned}
$$

Thus,

$$
\begin{aligned}
|\hat{f}(\omega)|< & +|f(1)||g(1)|+\left|f\left(t_{1}\right)\right||g(0)| \\
& +\sum_{k=1}^{m-1}\left|f\left(t_{k+1}\right)-f\left(t_{k}\right)\right|\left|g\left(t_{k}\right)\right| \\
\leq & +2 \frac{1}{2 \pi|\omega|}+\frac{1}{2 \pi} V(f) \frac{1}{|\omega|},
\end{aligned}
$$

since $f(x) \in[0,1], \forall x \in[0,1]$ and $|g(t)| \leq 1 /$ $(2 \pi|\omega|)$. Letting $\varepsilon \rightarrow 0$, we have obtained the desired result. 
Now we consider the one-dimensional dynamical system $(I, f)$. That is, we consider the dynamical behavior of the iterates $f^{(n)}$ of $f$ as $n \rightarrow \infty$.

For a given mother wavelet $\psi \in L^{2}(\mathbb{R})$, the continuous wavelet transform of $f^{(n)}$ with respect to $\psi$ is

$$
\begin{aligned}
W\left(f^{\circledR}\right)(s, b) & =\left\langle f^{\circledR}, \psi_{s, b}\right\rangle \\
& =\int_{\mathbb{R}} f^{\circledR}(t) \frac{1}{\sqrt{|s|}} \overline{\left(\frac{t-b}{s}\right)} d t .
\end{aligned}
$$

Here and in the following, we view $f^{\circledR}$ as a function on the whole real line $\mathbb{R}$ by setting $f^{\circledR}=0$ outside the interval $[0,1]$.

Theorem 1. Let $f: I \rightarrow I$ be continuous and piecewise monotone with finitely many extremal points and $\psi$ be a mother wavelet. In addition, if $\psi$ satisfies

$$
A_{\psi} \equiv \int_{\mathbb{R}} \frac{|\hat{\psi}(\omega)|}{|\omega|} d \omega<+\infty
$$

then

$$
\begin{aligned}
\sup _{s \neq 0, b \in \mathbb{R}}\left\{|s|^{-1 / 2}\left|W\left(f^{(n)}\right)(s, b)\right|\right\} \\
\quad \leq \frac{A_{\psi}}{2 \pi}\left(2+V\left(f^{(0)}\right)\right), \quad \forall n=1,2,3, \ldots .
\end{aligned}
$$

Proof. It suffices to prove that

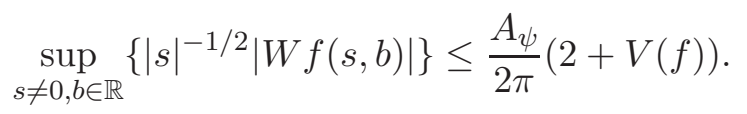

From the Parseval identity, we obtain

$$
\begin{aligned}
(W f)(s, b) & =\int_{\mathbb{R}} f \frac{1}{\sqrt{|s|}} \overline{\psi\left(\frac{t-b}{s}\right)} d t \\
& =\int_{\mathbb{R}} \hat{f}(\omega) \sqrt{|s|} \overline{\hat{\psi}(s \omega)} e^{i 2 \pi b \omega} d \omega .
\end{aligned}
$$

By Lemma 2 and (22), the above leads to

$$
\begin{aligned}
|(W f)(s, b)| & \leq \int_{\mathbb{R}}|\hat{f}(\omega)| \sqrt{|s|}|\hat{\psi}(s \omega)| d \omega \\
& \leq \int_{\mathbb{R}} \frac{2+V(f)}{2 \pi|\omega|} \sqrt{|s|}|\hat{\psi}(s \omega)| d \omega \\
& \leq \frac{\sqrt{|s|}}{2 \pi}(2+V(f)) \int_{\mathbb{R}} \frac{|\hat{\psi}(\omega)|}{|\omega|} d \omega \\
& =\frac{\sqrt{|s|} A_{\psi}}{2 \pi}(2+V(f)) .
\end{aligned}
$$

Therefore, we have established (24).
Replace $f$ in (24) with $f^{\circledR}$, we get (23).

Condition (22) can be eliminated, as shown in the following.

Corollary 3.1. Under the same assumptions as in Theorem 1 except (22), we have

$$
\begin{aligned}
& \sup _{s \neq 0, b \in \mathbb{R}}\left\{|s|^{-1 / 2}\left|W\left(f^{(\mathbb{B})}\right)(s, b)\right|\right\} \\
& \leq A\left(V\left(f^{\boxplus}\right)+1\right), \quad \forall n=1,2,3, \ldots,
\end{aligned}
$$

where

$$
A=\|\theta(t)\|_{\infty},
$$

and $\theta(t) \equiv \int_{0}^{t} \psi(\tau) d \tau$.

Proof. We follow the proof of [Boyarsky \& Góra, 1997, Lemma 2.3.2, p. 24] that for any interval $[c, d]$ in $\mathbb{R}$,

$$
\int_{c}^{d} g(t) \overline{\psi(t)} d t \leq\|\theta(t)\|_{\infty}\left(V_{[c, d]}(g)+\|g\|_{\infty}\right),
$$

where $g(t)=f(s t+b)$ and $V_{[c, d]}(g)$ denote the variation of $g$ on $[c, d]$. Thus,

$$
\begin{aligned}
|(W f)(s, b)| & =\left|\int_{\mathbb{R}} f \frac{1}{\sqrt{|s|}} \overline{\psi\left(\frac{t-b}{s}\right)} d t\right| \\
& =\left|\frac{s}{\sqrt{s}} \int_{-b / s}^{(1-b) / s} f(s \tau+b) \overline{\psi(\tau)} d \tau\right| \\
& \leq \sqrt{s}\|\theta(t)\|_{\infty}\left(V_{\left[-\frac{b}{s}, \frac{1-b}{s}\right]}(g)+\|g\|_{\infty}\right) \\
& \leq \sqrt{s}\|\theta(t)\|_{\infty}\left(V(f)+\|f\|_{\infty}\right) \\
& \leq \sqrt{s}\|\theta(t)\|_{\infty}(V(f)+1) .
\end{aligned}
$$

We thus obtain the result by using $f^{\circledR}$ for $f$.

Example 3.1. The Haar wavelet may be said to be the earliest wavelet, dating back to Haar's work in the early 20 th century. It is defined as

$$
\psi(t)= \begin{cases}1 \quad & 0 \leq t<\frac{1}{2}, \\ -1 \quad & \frac{1}{2} \leq t \leq 1,\left(=\chi_{[0,1 / 2)}(t)-\chi_{[1 / 2,1]}(t)\right), \\ 0 & \text { otherwise, }\end{cases}
$$

where $\chi_{J}$ denote the characteristic function of an interval $J$. We have

$$
\hat{\psi}(\omega)=\frac{1}{i 2 \pi \omega}\left(1-e^{-i \pi \omega}\right)^{2} .
$$


A direct computation shows that condition (22) is satisfied.

We will use Haar's wavelet shortly as a major example.

Example 3.2. For a family of derivatives of a Gaussian wavelet

$$
\psi(t)=\frac{(-1)^{m+1}}{\sqrt{\Gamma\left(\frac{m+1}{2}\right)}} \frac{d^{m}}{d t^{m}}\left(e^{-t^{2} / 2}\right),
$$

by direct computation, one obtains

$$
\hat{\psi}(\omega)=\frac{-i^{m}}{\sqrt{\Gamma\left(\frac{m+1}{2}\right)}} \omega^{m} e^{-\frac{\omega^{2}}{2}}, \quad m=1,2, \ldots
$$

Thus, again, condition (22) holds for $m=1,2, \ldots$.

From Theorem 1, we obtain a criterion for chaos by wavelet transform.

Corollary 3.2. Under the assumptions of Theorem 1 , if there exist $s_{n} \neq 0$ and $b_{n} \in \mathbb{R}$ such that

$$
\lim _{n \rightarrow+\infty} \frac{1}{n} \ln \left|s_{n}\right|^{-1 / 2}\left|W\left(f^{\circledR}\right)\left(s_{n}, b_{n}\right)\right|>0,
$$

then

$$
\lim _{n \rightarrow+\infty} \frac{1}{n} \ln V\left(f^{\circledR}\right)>0 .
$$

Thus, the system $(I, f)$ has positive topological entropy and is chaotic in the sense of Li-Yorke.

Proof. The first part comes directly from Theorem 1. The second part follows from the first part and the results in [Chen et al., 2011; Chen et al., 2004].

Without condition (22), instead, if we assume that $\left\{\psi_{j, k} ; j, k \in Z\right\}$ forms an orthonormal wavelet basis for $L^{2}(\mathbb{R})$ which is derivable by Lemma 2 , we can establish not only necessary conditions but also sufficient conditions for chaos of the system $(I, f)$ in terms of the corresponding wavelet coefficients. There are two ways to decompose $f$ in terms of the basis for $L^{2}(\mathbb{R})$. One is to extend $f$ as a function on the whole line $\mathbb{R}$ by setting $f(t)=0$ outside the interval $[0,1]$ and then decompose it as (13) or (14). This approach is often not appreciated from the point of view of wavelet analysis since it introduces an artificial "jump" at the boundary of $I$, and is reflected in the wavelet coefficients. Another is to modify the orthonormal basis for $L^{2}(\mathbb{R})$ such that it forms an orthonormal basis for $L^{2}(0,1)$ and then decompose $f$ in terms of the modified basis. There are several different ways to achieve this modification. But the basis for $L^{2}(0,1)$ obtained becomes more complicated and as a result it is difficult to compute the corresponding wavelet coefficients analytically. Here, since we are concerned only with the dynamics, the artificial jump at the boundary caused by the former approach does not affect our analysis. Therefore, we have decided to take the extension by setting $f=0$ outside the interval $I$.

By Lemma 2, $f$ has decomposition (13) or (14). In addition, if the scaling function $\varphi$ and the mother function $\psi$ have compact supports, which, without loss of generality, we may assume that they are contained in $(0,1)$, then for any given $j_{0}>0, j \geq j_{0}$ and $n \geq 2^{j}$, the support of $\psi_{j, n}$ has no intersection with $[0,1]$. Nor does $\varphi_{j_{0}, n}$ for $n \geq 2^{j_{0}}$. Thus decomposition (14) can be written as

$f(t)=\sum_{j \geq j 0} \sum_{k=0}^{2^{j}-1} d_{j, k} \psi_{j, k}(t)+\sum_{k=0}^{2^{j_{0}}-1} c_{j_{0}, k} \varphi_{j_{0}, k}(t)$,

for any $j_{0}>0$.

Theorem 2. Let $\varphi$ and $\psi$ be real and have compact supports in $[0,1]$. If $f \in W^{1,1}(0,1)$, then there exists a constant $A>0$ such that

$$
V(f) \geq A \sup _{j \geq j_{0}} 2^{j / 2} \sum_{k=0}^{2^{j}-1}\left|\left\langle f, \psi_{j, k}\right\rangle\right| .
$$

Proof. Let

$$
\theta(t)=\int_{0}^{t} \psi(\tau) d \tau
$$

Then from (5) $\theta$ has support in $[0,1]$. For any $j \geq j_{0}$ doing integration by parts, we have

$$
\begin{aligned}
& \sum_{k=0}^{2^{j}-1}\left|\left\langle f, \psi_{j, k}\right\rangle\right| \\
& \quad=\sum_{k=0}^{2^{j}-1}\left|\int_{0}^{1} f(t) 2^{j / 2} \psi\left(2^{j} t-k\right) d t\right| \\
& =\sum_{k=0}^{2^{j}-1}\left|\int_{0}^{1} f^{\prime}(t) 2^{-j / 2} \theta\left(2^{j} t-k\right) d t\right| \\
& \leq 2^{-j / 2} \sum_{k=0}^{2^{j}-1}\left|\int_{0}^{1}\right| f^{\prime}(t)|| \theta\left(2^{j} t-k\right) d t \mid .
\end{aligned}
$$


Since the support of $\theta$ is contained in [0,1], it follows that

$$
\begin{aligned}
\sum_{k=0}^{2^{j}-1}\left|\left\langle f, \psi_{j, k}\right\rangle\right| & \leq 2^{-j / 2} \sup _{t \in \mathbb{R}}|\theta(t)| \int_{0}^{1}\left|f^{\prime}(t)\right| d t \\
& =2^{-j / 2} A^{-1} \underbrace{\int_{0}^{1}\left|f^{\prime}(t)\right| d t}_{V(f)},
\end{aligned}
$$

where $A^{-1}=\sup _{t \in \mathbb{R}}|\theta(t)|$. Thus we have $(27)$.

Theorem 3. Let $\varphi$ and $\psi$ have compact supports in $[0,1]$. If, in addition, $V(\psi)<+\infty$, then there exists a constant $B \geq 1$ such that

$$
\begin{aligned}
V(f) \leq B & {\left[\sum_{j \geq j_{0}} \sum_{k=0}^{2^{j}-1} 2^{j / 2}\left|\left\langle f, \psi_{j, k}\right\rangle\right|\right.} \\
& \left.+2^{j_{0} / 2} \sum_{k=0}^{2^{j_{0}}-1} \mid\left\langle f, \varphi_{j_{0}, k}\right\rangle\right] .
\end{aligned}
$$

Proof. It follows from (26) that

$$
\begin{aligned}
V(f) \leq & \sum_{j \geq j_{0}} \sum_{k=0}^{2^{j}-1}\left|\left\langle f, \psi_{j, k}\right\rangle\right| V\left(\psi_{j, k}\right) \\
& +\sum_{k=0}^{2^{j_{0}}-1}\left|\left\langle f, \varphi_{j_{0}, k}\right\rangle\right| V\left(\varphi_{j_{0}, k}\right),
\end{aligned}
$$

in which

$$
V\left(\psi_{j, k}\right)=\int_{0}^{1} 2^{j / 2} 2^{j}\left|\psi^{\prime}\left(2^{j}-k\right)\right| d t \leq 2^{j / 2} V(\psi) .
$$

And similarly,

$$
V\left(\varphi_{j_{0}, k}\right) \leq 2^{j_{0} / 2} V(\varphi) .
$$

The inequality (28) follows from the above three inequalities.

Corollary 3.3. Assume that $\varphi$ and $\psi$ have compact supports in $[0,1]$ with finite total variations.

(1) If $f \in W^{1, \infty}$ and there exists an increasing integer sequence $j_{n} \rightarrow \infty$ and

$$
\lim _{n \rightarrow \infty} \frac{1}{n} \ln \left(2^{j_{n} / 2} \sum_{k=0}^{2^{j_{n}}-1}\left|\left\langle f^{\circledR}, \psi_{j_{n}, k}\right\rangle\right|\right)>0,
$$

then

$$
\lim _{n \rightarrow \infty} \frac{1}{n} \ln V\left(f^{(n)}\right)>0,
$$

and $f$ has chaotic oscillations.
(2) If $f \in W^{1, \infty}$ and there exists an increasing integer sequence $j_{n} \rightarrow+\infty$ such that

$$
\lim _{n \rightarrow \infty} \sum_{k=0}^{2^{j_{n}}-1}\left|\left\langle f^{\circledR}, \psi_{j_{n}, k}\right\rangle\right|>0
$$

then

$$
\lim _{n \rightarrow \infty} \frac{1}{n} \ln V\left(f^{(n)}\right)>0,
$$

and $f$ has chaotic oscillations.

(3) Conversely, if $f \in W^{1, \infty}$ and

$$
\lim _{n \rightarrow \infty} \frac{1}{n} \ln V\left(f^{(n)}\right)>0,
$$

then

$$
\begin{aligned}
& {\left[\sum_{j \geq j_{0}} \sum_{k=0}^{2^{j}-1} 2^{j / 2}\left|\left\langle f^{\circledR}, \psi_{j, k}\right\rangle\right|\right.} \\
& \left.\quad+2^{j_{0} / 2} \sum_{k=0}^{2^{j_{0}-1}}\left|\left\langle f^{\circledR}, \varphi_{j_{0}, k}\right\rangle\right|\right]
\end{aligned}
$$

grows exponentially as $n \rightarrow \infty$.

Example 3.3. Consider the tent map (as in Part I [Chen et al., 2011]):

$$
f(t)= \begin{cases}2 t & \text { if } 0 \leq t<\frac{1}{2} \\ -2(t-1) & \text { if } \frac{1}{2} \leq t \leq 1\end{cases}
$$

This map is well known to be chaotic. Here we apply Corollary 3.3 part (1) to this map. We choose the Haar wavelet as in Example 3.1:

$$
\psi(t)=\chi_{\left[0, \frac{1}{2}\right)}(t)-\chi_{\left[\frac{1}{2}, 1\right]}(t) .
$$

For $j>0$, consider the wavelet coefficients of $f^{\circledR}$, where by Part I [Chen et al., 2011],

$$
f^{\circledR}(t)= \begin{cases}2^{n} t-2(l-1), & \text { if } \frac{2(l-1)}{2^{n}} \leq t<\frac{2 l-1}{2^{n}}, \\ -2^{n} t+2 l, & \text { if } \frac{2 l-1}{2^{n}} \leq t \leq \frac{2 l}{2^{n}},\end{cases}
$$

$l=1,2, \ldots, 2^{n-1}$. 
For $k=0,1, \ldots, 2^{j}-1$, we compute the wavelet coefficients of $f^{(\pi)}$ with respect to the wavelet basis $\left\{\psi_{j, k}\right\}$ :

$$
\begin{aligned}
d_{j, k}^{n} & \equiv \int_{0}^{1} f^{\boxplus}(t) \psi_{j, k}(t) d t \\
& =2^{j / 2} \int_{0}^{1} f^{\boxplus}(t) \psi\left(2^{j} t-k\right) d t \\
& =2^{-j / 2} \int_{-k}^{2^{j}-k} f^{\boxplus}\left(2^{-j}(\tau+k)\right) \psi(\tau) d \tau \\
& =2^{-j / 2} \int_{0}^{1} f^{\circledR}\left(2^{-j}(\tau+k)\right) \psi(\tau) d \tau,
\end{aligned}
$$

by change of variables and noting that $\operatorname{supp}(\psi)=$ $[0,1]$, where "supp" means "the support of". Substituting (29) into the above equation, we have

$$
\begin{aligned}
d_{j, k}^{n}=2^{j / 2} & {\left[\int_{2^{-j} k}^{2^{-j}(k+1 / 2)} f^{\circledR}(t) d t\right.} \\
& \left.-\int_{2^{-j}(k+1 / 2)}^{2^{-j}(k+1)} f^{\circledR}(t) d t\right] .
\end{aligned}
$$

Taking $j=n$, from (30), it follows that when $k$ is even

$$
\begin{aligned}
d_{n, k}^{n}= & 2^{n / 2}\left[\int_{2^{-n} k}^{2^{-n}(k+1 / 2)}\left(2^{n} t-k\right) d t\right. \\
& \left.\quad-\int_{2^{-n}(k+1 / 2)}^{2^{-n}(k+1)}\left(2^{n} t-k\right) d t\right] \\
= & 2^{n / 2} 2^{-(n+1)}\left(-\frac{1}{2}\right)
\end{aligned}
$$

and when $k$ is odd,

$$
\begin{aligned}
d_{n, k}^{n}= & 2^{n / 2}\left[\int_{2^{-n} k}^{2^{-n}(k+1 / 2)}\left(-2^{n} t+k\right) d t\right. \\
& \left.\quad-\int_{2^{-n}(k+1 / 2)}^{2^{-n}(k+1)}\left(-2^{n} t+k\right) d t\right] \\
= & 2^{n / 2} 2^{-(n+1)}\left(\frac{1}{2}\right) .
\end{aligned}
$$

Therefore

$$
\sum_{k=0}^{2^{n}-1}\left|d_{n, k}^{n}\right|=2^{\frac{n}{2}-2}>0
$$

which, by Corollary 3.3, implies

$$
\lim _{n \rightarrow \infty} \frac{1}{n} \ln V\left(f^{(n)}\right)>0 .
$$

In fact, combining with Theorem 2, we have

$$
\lim _{n \rightarrow \infty} \frac{1}{n} \ln V\left(f^{(n)}\right) \geq \ln 2 .
$$

\section{The Growth Rate of the Total Variation of $f^{\circledR}$ in Terms of the Wavelet Coefficient of the Haar Wavelet}

Let $f:[0,1] \rightarrow[0,1]$ be continuous and piecewise monotone, and $\psi$ be a mother wavelet with compact supports in $[0,1]$. For $j \geq j_{0}$ with respect to the scaling function $\varphi$, denote by $d_{j, k}^{n}$ the wavelet coefficients of $f^{\circledR}$. That is,

$$
d_{j, k}^{n}=\left\langle f^{\circledR}, \psi_{j, k}\right\rangle=\int_{0}^{1} f^{\circledR}(t) 2^{j / 2} \psi\left(2^{j} t-k\right) d t .
$$

Then by Theorem 2, we have

$$
V\left(f^{(n)}\right) \geq A \sup _{j \geq j_{0}} 2^{j / 2} \sum_{k=0}^{2^{j}-1}\left|d_{j, k}^{n}\right|,
$$

where $A>0$ is defined in Theorem 2 .

We denote by $\tilde{d}_{j, k}^{n}$ the coefficients of $f$ under the basis $\left\{\tilde{\psi}_{j, k}(t) \equiv 2^{j / 2} \psi\left(2^{j} t-k-(1 / 2)\right)\right\}_{j, k \in Z}$. That is, for $k=0,1, \ldots, 2^{j}-2$,

$$
\begin{aligned}
\tilde{d}_{j, k}^{n} & =\int_{0}^{1} f(n) 2^{j / 2} \psi\left(2^{j} t-k-\frac{1}{2}\right) d t, \\
\tilde{d}_{j, 2^{j}-1}^{n} & =0 .
\end{aligned}
$$

Please note that for $k \geq 2^{j}-1$ the support of $\tilde{\psi}_{j, k}$ has empty intersection with $[0,1]$.

By the same approach as in the proof of Theorem 2, we also have

$$
V\left(f^{\circledR}\right) \geq A \sup _{j \geq j_{0}} 2^{j / 2} \sum_{k=0}^{2^{j}-1}\left|\tilde{d}_{j, k}^{n}\right| .
$$

If we denote

$$
\mathcal{W}_{\psi}\left(f^{\circledR}\right)=\lim _{j \rightarrow \infty} 2^{j / 2} \sum_{k=0}^{2^{j}-1}\left(\left|d_{j, k}^{n}\right|+\left|\tilde{d}_{j, k}^{n}\right|\right),
$$

then from (32) and (33), we obtain

$$
2 V\left(f^{\circledR}\right) \geq A \mathcal{W}_{\psi}\left(f^{\circledR}\right) .
$$


Thus we have

$$
\lim _{n \rightarrow \infty} \frac{1}{n} \ln V\left(f^{\boxplus}\right) \geq \lim _{n \rightarrow \infty} \frac{1}{n} \ln \mathcal{W}_{\psi}\left(f^{(n)}\right) .
$$

A natural question here is if there exists a wavelet such that inequality (35) becomes equality. We have the following.

Theorem 4. Let $f:[0,1] \rightarrow[0,1]$ be continuous and piecewise monotone, and $\psi$ be the Haar wavelet given by (29). Then

$$
\lim _{n \rightarrow \infty} \frac{1}{n} \ln V\left(f^{\boxplus}\right)=\lim _{n \rightarrow \infty} \frac{1}{n} \ln \mathcal{W}_{\psi}\left(f^{(n)}\right),
$$

where $\mathcal{W}_{\psi}\left(f^{\circledR}\right)$ is defined by (34).

Proof. Since the mother wavelet we choose here is the Haar wavelet, we have (31). Thus

$$
\begin{array}{r}
d_{j, k}^{n}=2^{j / 2}\left[\int_{2^{-j} k}^{2^{-j}(k+1 / 2)} f^{\boxplus}(t) d t\right. \\
\left.-\int_{2^{-j}(k+1 / 2)}^{2^{-j}(k+1)} f^{\boxplus}(t) d t\right] \\
=2^{j / 2}\left(f^{\boxplus}\left(\xi_{k}\right)-f^{\boxplus}\left(\xi_{k}^{\prime}\right)\right) 2^{-j-1}, \\
k=0,1, \ldots, 2^{j}-1,
\end{array}
$$

for some

$$
\begin{aligned}
& \xi_{k} \in\left[2^{-j} k, 2^{-j}\left(k+\frac{1}{2}\right)\right], \\
& \xi_{k}^{\prime} \in\left[2^{-j}\left(k+\frac{1}{2}\right), 2^{-j}(k+1)\right] .
\end{aligned}
$$

By the same argument, we have for the coefficients $\tilde{d}_{j, k}^{n}$,

$$
\begin{array}{r}
\tilde{d}_{j, k}^{n}=2^{j / 2}\left[\int_{2^{-j}(k+1 / 2)}^{2^{-j}(k+1)} f^{\boxplus}(t) d t\right. \\
\left.-\int_{2^{-j}(k+1)}^{2^{-j}(k+3 / 2)} f^{\circledR}(t) d t\right] \\
=2^{j / 2}\left(f^{\circledR}\left(\xi_{k}^{\prime}\right)-f^{\circledR}\left(\xi_{k+1}\right)\right) 2^{-j-1}, \\
k=0,1, \ldots, 2^{j}-2 .
\end{array}
$$

Therefore

$$
\begin{aligned}
2^{j / 2}\left[\sum_{k=0}^{2^{j}-1}\left|d_{j, k}^{n}\right|+\sum_{k=0}^{2^{j}-2}\left|\tilde{d}_{j, k}^{n}\right|\right] \\
=\frac{1}{2}\left[\sum_{k=0}^{2^{j}-1}\left|f^{\circledR}\left(\xi_{k}\right)-f^{\circledR}\left(\xi_{k}^{\prime}\right)\right|\right. \\
\left.\quad+\sum_{k=0}^{2^{j}-2}\left|f^{\circledR}\left(\xi_{k}^{\prime}\right)-f^{\circledR}\left(\xi_{k+1}\right)\right|\right] \\
\rightarrow \frac{1}{2} V\left(f^{\boxplus n}\right), \quad \text { as } j \rightarrow \infty .
\end{aligned}
$$

We have (36) and the proof is complete.

Remark 4.1. It follows from Theorem 4 that a onedimensional dynamical system $(I, f)$ has chaotic behavior if and only if there exists an orthonormal wavelet basis (Haar wavelet) $\left\{\psi_{j, k}\right\}_{j, k \in Z}$ such that either

$$
2^{j_{n} / 2}\left[\sum_{k=0}^{2^{j_{n}}-1}\left|d_{j_{n}, k}^{n}\right|\right]
$$

or

$$
2^{j_{n} / 2}\left[\sum_{k=0}^{2^{j_{n}}-1}\left|\tilde{d}_{j_{n}, k}^{n}\right|\right]
$$

grows exponentially as $j_{n} \rightarrow \infty$.

Remark 4.2. It is well known that the Haar wavelet is disadvantageous in wavelet analysis since it lacks smoothness. Here, rather, we see from Theorem 4 that for interval maps Haar's wavelet can be efficiently used to detect the growth of the total variation of iterates of $f$.

Finally, we give a result pertaining to topological conjugate systems.

Theorem 5. Let $f$ and $g$ be continuous maps from $I$ into itself and be piecewise monotone, and $\psi$ be the Haar wavelet. If $f$ and $g$ have topological conjugacy, then

$$
\lim _{n \rightarrow \infty} \frac{1}{n} \ln \mathcal{W}_{\psi}\left(f^{(n)}\right)=\lim _{n \rightarrow \infty} \frac{1}{n} \ln \mathcal{W}_{\psi}\left(g^{\circledR}\right) .
$$

In other words, the quantity

$$
\lim _{n \rightarrow \infty} \frac{1}{n} \ln \mathcal{W}_{\psi}\left(f^{(n)}\right)
$$

is a topological invariant. 
Proof. This follows from Theorem 4 and the fact that

$$
\lim _{n \rightarrow \infty} \frac{1}{n} \ln V\left(f^{(n)}\right)=\lim _{n \rightarrow \infty} \frac{1}{n} \ln V\left(g^{(n)}\right),
$$

since $f$ and $g$ are topologically conjugate and piecewise monotone.

We remark here that the basis $\left\{\tilde{\psi}_{j, k}(t) \equiv\right.$ $\left.2^{j / 2} \psi\left(2^{j} t-k-(1 / 2)\right)\right\}_{j, k \in Z}$ defined above may not be a wavelet basis. So the corresponding coefficients $\tilde{d}_{j, k}^{n}$ cannot be taken as a wavelet coefficient. In the last part of the paper, we establish the wavelet coefficients $d_{j, k}^{n}$ related to the Lyapunov exponent of one-dimensional dynamical systems. More importantly, we can estimate the wavelet coefficient $d_{j, k}^{n}$ of $f^{\circledR}$ from some properties of $f$, but not from its iterates $f^{\circledR}$.

Let $f:[0,1] \rightarrow[0,1]$ be $C^{1}$ and

$$
\left|f^{\prime}(x)\right| \leq L
$$

except at a finite number of points and $\psi$ be the Haar wavelet given by (29). Then for any $j=0$, $1,2, \ldots$ and $k=0,1,2, \ldots, 2^{j}-1$, we have

$$
\begin{aligned}
d_{j, k}^{n}= & 2^{j / 2}\left[\int_{2^{-j} k}^{2^{-j}(k+1 / 2)} f^{\circledR}(t) d t\right. \\
& \left.\quad-\int_{2^{-j}(k+1 / 2)}^{2^{-j}(k+1)} f^{\circledR}(t) d t\right] \\
= & 2^{j / 2} \int_{2^{-j} k}^{2^{-j}(k+1 / 2)}\left(f^{\circledR}(t)-f^{\circledR}\left(t+2^{-j-1}\right)\right) d t \\
= & 2^{-(j / 2)-1} \int_{2^{-j} k}^{2^{-j}(k+1 / 2)} f^{\circledR \prime}(\tau(t)) d \tau,
\end{aligned}
$$

for some

$$
\tau(t) \in\left[2^{-j} k, 2^{-j}(k+1)\right] .
$$

Thus

$$
\left|d_{j, k}^{n}\right| \leq 2^{-j / 2-1} L^{n} 2^{-j-1}, \quad 2^{3 j / 2}\left|d_{j, k}^{n}\right| \leq \frac{1}{4} L^{n} .
$$

Recall that for a piecewise smooth map $f$ : $[0,1] \rightarrow[0,1]$, and $t_{0} \in[0,1]$ such that $f^{\circledR \prime}\left(t_{0}\right)$ is well-defined for $n \in Z_{+}$, then the quantity

$$
\lambda\left(t_{0}\right)=\limsup _{n \rightarrow \infty} \frac{1}{n} \ln \left|f^{\boxplus \prime}\left(t_{0}\right)\right|
$$

is said to be the Lyapunov exponent of $t_{0}$. Let $\mu$ be a Borel probability $f$-invariant ergodic measure. Then, for every $\mu$-typical point $t_{0}$ (i.e. it belongs to a set of $\mu$-measure one), we have

$$
\lambda\left(t_{0}\right)=\int_{0}^{1} \ln \left|f^{\prime}(x)\right| d \mu,
$$

by the Birkhoff Ergodic theorem. Furthermore, if $\mu$ is an absolutely continuous invariant measure, that is, there exists a density function $\rho(\cdot)$ such that

$$
\mu(A)=\int_{A} \rho(x) d x
$$

for any measurable set $A$, and $\rho(x) \neq 0, \mathcal{L}$-a.e. $x \in[0,1]$. Here $\mathcal{L}$ denotes the Lebesgue measure. Then the set of all $\mu$-typical points has full-measure with respect to the Lebesgue measure. Therefore, from Eq. (37), for any $j=0,1,2, \ldots$, and $k=$ $0,1,2, \ldots, 2^{j}-1$

$$
2^{3 j / 2}\left|d_{j, k}^{n}\right| \leq \frac{1}{4} \lambda^{n}
$$

for $n$ large enough. Summarizing this, we have

Theorem 6. Let $f:[0,1] \rightarrow[0,1]$ be a piecewise smooth map and have absolutely continuous invariant measure with the density function being nonzero for $\mathcal{L}$-almost all points in $[0,1]$. If $f$ has negative Lyapunov exponent, then

$$
2^{3 j / 2}\left|d_{j, k}^{n}\right| \rightarrow 0, \quad n \rightarrow \infty
$$

uniformly for $j=0,1,2, \ldots$ and $k=0,1,2, \ldots$, $2^{j}-1$

For the case when the Lyapunov exponent is positive, we have

Theorem 7. Let a continuous map $f:[0,1] \rightarrow[0,1]$ be piecewise expanding. That is, there is $\lambda>1$ such that $\left|f^{\prime}\right| \geq \lambda$, except at a finite number of points. Then for any positive integer $n$, there exists $j_{n}$ such that for $j>j_{n}$ there is at least an $k \in$ $\left\{0,1,2, \ldots, 2^{j}-1\right\}$ with

$$
2^{3 j / 2}\left|d_{j, k}^{n}\right| \geq \frac{1}{4} \lambda^{n} .
$$

Remark 4.3. The Lasota-Yorke theorem implies that every $C^{2}$ piecewise expanding map has an absolutely continuous invariant measure [Lasota \& Yorke, 1973]. Such kind of maps also have positive Lyapunov exponent. These indicate the presence of 
dynamical chaos. Theorem 7 implies the quantity $2^{3 j / 2}\left|d_{j, k}^{n}\right|$ grows exponentially as $n \rightarrow \infty$.

Proof of Theorem 7. Let $l(f)$ denote the lap number of $f$ and $s$ the growth number of $f$ [Milnor \& Thurston, 1988]. That is

$$
s=\lim _{n \rightarrow \infty} l\left(f^{\boxplus n}\right)^{1 / n}=\inf _{n \geq 1} l\left(f^{(n)}\right)^{1 / n} .
$$

It is easy to see that $s \in[\lambda, l(f)]$. Actually the logarithm of $s$ is equal to the topological entropy of $f$. See, for example, [Milnor \& Thurston, 1988].

It follows from (39) that there exists $N$ such that when $n>N$,

$$
(s+1)^{n} \geq l\left(f^{(n)}\right) .
$$

Let $m$ be such that

$$
2^{m}>\max \left\{l(f), l\left(f^{2}\right), \ldots, l\left(f^{N}\right), s+1\right\} .
$$

Let $j_{n}=m n$. Then when $j>j_{n}$, we have

$$
2^{j}>l\left(f^{(n)}\right)
$$

Recall that $l\left(f^{\circledR}\right)$ is the lap number of $f^{\circledR}$. So there is at least an interval $\left[2^{-j} k, 2^{-j}(k+1)\right]$ on which $f^{(n)}$ is strictly monotone for some $k \in\{0,1,2, \ldots$, $\left.2^{j}-1\right\}$. Thus

$$
\begin{aligned}
2^{3 j / 2}\left|d_{j, k}^{n}\right| & \geq 2^{j-1} \int_{2^{-j} k}^{2^{-j}(k+1 / 2)}\left|f^{囚 \prime}(\tau(t))\right| d \tau \\
& \geq \frac{1}{4} \lambda^{n},
\end{aligned}
$$

by (37).

A time series $f^{\circledR}$ generated by a deterministic chaotic interval map $f$ is known to behave chaotically on some subintervals while nonchaotically on other subintervals. In this case, the use of wavelet analysis would offer distinct advantages over Fourier analysis due to certain wavelets' multiresolution capability. Nevertheless, concrete examples with explicitly calculated wavelet coefficients are nearly impossible to construct due to the complexities involved. Thus, one must still rely on numerics to perform simulations.

\section{Acknowledgments}

Goong Chen is supported in part by the Texas Advanced Research Project (ARP) Grant No. 010336-0149-2009 from THECB and Qatar National Research Fund (QNRF) Grant No. NPRP 09-462-1-074. Sze-Bi is supported in part by grants from the National Science Council and the Ministry of Education of Republic of China. Yu Huang is supported in part by the National Natural Science Foundation of China (Grants 10771222 and 11071263) and the NSF of Guangdong Province.

\section{References}

Azad, S. \& Sett, S. K. [2003] "Wavelet detecting chaos in logistic map," Natl. Conf. Nonlinear Syst. Dyn., Indian Institute of Technology, Kharagpur 721302, pp. 301-304.

Boyarsky, A. \& Góra, P. [1997] Laws of Chaos: Invariant Measures and Dynamical Systems in One Dimension (Birkhäuser).

Chen, G., Hsu, S. B., Huang, Y. \& Roque-Sol, M. [2011] "The spectrum of chaotic time series (I): Fourier analysis," Int. J. Bifurcation and Chaos 21, 1439-1456.

Chen, G., Huang, T. \& Huang, Y. [2004] "Chaotic behavior of interval maps and total variations of iterates," Int. J. Bifurcation and Chaos 14, 2161-2186.

Dai, D. D. [2006] Lectures on Wavelets (Zhongshan (Sun Yat-Sen) University (in Chinese)).

Daubechies, I. [1992] Ten Lectures on Wavelets (SIAM, Philadelphia, PA).

Edwards, R. E. [1979] Fourier Series a Modern Introduction, 2nd edition (Springer-Verlag, NY-BerlinHeidelberg).

Lasota, A. \& Yorke, J. A. [1973] "On the existence of invariant measures, for piece-wise monotonic transformations," Trans. Amer. Math. Soc. 186, 481-488.

Meyer, Y. \& Salinger, D. H. [1995] Wavelets and Operators, Cambridge Studies in Advanced Mathematics, Vol. 37 (Cambridge, UK).

Milnor, J. \& Thurston, W. [1988] "On iterated maps of interval," Dynamical Systems: Proc. 1986-87, Lecture Notes in Mathematics, Vol. 1342 (Springer-Verlag), pp. $463-563$.

Misiurewicz, M. [2004] "On Bowen's definition of topological entropy," Discr. Contin. Dyn. Syst. 10, 827833.

Perman, D. \& Hamilton, I. [1992] "Wavelet analysis of time series for the Duffing oscillator: The detection of order within chaos," Phys. Rev. Lett. 69, 2607-2610. 